University of Wollongong

Research Online

Faculty of Informatics - Papers (Archive)

Faculty of Engineering and Information

Sciences

1975

\title{
On the matrices used to construct Baumert-Hall arrays
}

Richard B. Lakein

Jennifer Seberry

University of Wollongong, jennie@uow.edu.au

Follow this and additional works at: https://ro.uow.edu.au/infopapers

Part of the Physical Sciences and Mathematics Commons

\section{Recommended Citation}

Lakein, Richard B. and Seberry, Jennifer: On the matrices used to construct Baumert-Hall arrays 1975. https://ro.uow.edu.au/infopapers/968

Research Online is the open access institutional repository for the University of Wollongong. For further information contact the UOW Library: research-pubs@uow.edu.au 


\title{
On the matrices used to construct Baumert-Hall arrays
}

\author{
Abstract \\ Four circulant (or type 1) $(0,1,-1)$ matrices $X_{1}, X_{2}, X_{3}, X_{4}$ of order $t$ with the property that each of the $t^{2}$ \\ positions is non-zero in precisely one of the $X_{i}$ and such that \\ $\mathrm{X}_{1} \mathrm{X}_{1}^{\mathrm{T}}+\mathrm{X}_{2} \mathrm{X}_{2}^{\mathrm{T}}+\mathrm{X}_{3} \mathrm{X}_{3}^{\mathrm{T}}+\mathrm{X}_{4} \mathrm{X}_{4}^{\mathrm{T}}=\mathrm{t} \mathrm{l}_{\mathrm{t}}$ \\ will be called T-matrices. \\ This paper studies the construction, use and properties of T-matrices giving a new construction for \\ Hadamard matrices and some new equivalence results for Hadamard matrices and Baumert-Hall arrays. \\ Disciplines \\ Physical Sciences and Mathematics \\ Publication Details \\ Richard B. Lakein and Jennifer Seberry Wallis, On the matrices used to construct Baumert-Hall arrays, \\ Combinatorics: III, Lecture Notes in Mathematics, 452, Springer--Verlag, Berlin--Heidelberg--New York, \\ (1975), 156-170.
}




\section{ON THE MATRICES USED TO CONSTRUCT BAUMERT-HALL ARRAYS}

\section{RICHARD B, LAKEIN AND JENNIFER SEBERRY WALLIS*}

Four circulant (or type 1) $(0,1,-1)$ matrices $x_{1}, x_{2}, x_{3}, x_{4}$ of order $t$ with the property that each of the $t^{2}$ positions is nonzero in precisely one of the $x_{i}$ and such that

$$
x_{1} x_{1}^{T}+x_{2} x_{2}^{T}+x_{3} x_{3}^{T}+x_{4} x_{4}^{T}=t I_{t}
$$

will be called T-matrices.

This paper studies the construction, use and properties of T-matrices giving a new construction for Hadamard matrices and some new equivalence results for Hadamard matrices and Baumert-Hall arrays.

\section{INTRODUCTION}

An Hadamard matrix $\mathrm{H}=\left(\mathrm{h}_{i j}\right)$ is a square matrix of order $\mathrm{n}$ with elements +1 or -1 which satisfies the matrix equation

$$
H^{T}=H^{T} H=n I_{n} \text {, }
$$

where $H^{T}$ denotes $H$ transposed and $I$ the identity matrix.

Unless specifically stated the order of matrices should be determined from the context. We use - for -1 and $J$ for the matrix with every element +1 .

The matrices

$$
[1],\left[\begin{array}{cc}
1 & 1 \\
1 & -
\end{array}\right],\left[\begin{array}{cccc}
- & 1 & 1 & 1 \\
1 & - & 1 & 1 \\
1 & 1 & - & 1 \\
1 & 1 & 1 & -
\end{array}\right],\left[\begin{array}{cccc}
1 & 1 & 1 & 1 \\
1 & - & 1 & - \\
1 & 1 & - & - \\
1 & - & - & 1
\end{array}\right]
$$

are Hadamard matrices of order 1, 2, 4 and 4 respectively.

It can be shown (see [7] and [20]) that the order of an Hadamard matrix is necessarily 1,2 or $4 m$ for some $m=1,2,3, \ldots$. It has

* Written while this author was visiting the Mathematics Department, S.U.N.Y. at Buffalo, New York. 
been conjectured that Hadamard matrices of all hese orders exist. For many years the first few unresolved cases hye been 183, 236 , 268 and 292. Richard $J$. Turyn has announced [1] that he has found Hadamard matrices for the orders 188 and 236. Euward Spence has announced [10] the existence of the matrix of orden 292. So thr 1iral few unresolved cases are now 268,412 and 428 .

The book [20] of Wallis, street and Wallis gives all the constructions for Hadamard matrices known to one of us early in 1972 but many exciting results have been discovered more recently. For many elementary definitions we refer the reader to this book.

In 1944 Williamson [24] introduced a special type of Hadamand matrix

$$
H=\left[\begin{array}{rrrr}
A & B & C & D \\
-B & A & -D & C \\
-C & D & A & -B \\
-D & -C & B & A
\end{array}\right]
$$

based on the matrix representation of the quaternions.

Subsequently four $(1,-1)$ matrices $A, B, C, D$ of order $m$ which satisfy

and

$$
\begin{aligned}
& \text { (i) } X Y^{T}=Y X^{T}, \quad X, Y \in\{A, B, C, D\}, \\
& \text { (ii) } A A^{T}+B B^{T}+C C^{T}+D D^{T}=4 m I_{m}
\end{aligned}
$$

have been called Williamson matrices. These matrices have been the subject of much recent study [8], [11], [12], [16], [17], [18], [22], [23], because

Theorem 1.1. If there exist williamson matrices of order $m$ then there exists an Hadamard matrix of order $4 \mathrm{~m}$.

Definition 1.2. The rows or columns of an array of indeterminates will be said to be formaly orthogonal if realizing the indeterminates as any elements from a commutative ring causes the distinct rows or columns of the array to be pairwise orthogonal.

Baumert and Hall (see [1]) in 1965 published a $12 \times 12$ array containing precisely $3 \pm A^{\prime} s, 3 \pm B^{\prime} s, 3 \pm C^{\prime} s, 3 \pm D^{\prime} s$ in each row and column. Furthermore the rows and columns were formally orthogonal. If the $A, B, C, D$ are matrices which pairwise satisfy $X Y^{T}=Y X^{T}$ then

$$
H H^{T}=I_{12} \times 3\left(A A^{T}+B B^{T}+C C^{T}+D D^{T}\right) .
$$


More generally we consider

Definition 1.3. A $4 \mathrm{t} \times 4 \mathrm{t}$ array of the indeterminates $\pm \mathrm{A}, \pm \mathrm{B}$, $\pm \mathrm{C}, \pm \mathrm{D}$ in which

(i) each indeterminate, $\pm x$, occurs precisely $t$ times in each row and column, and

(ii) the distinct rows are formally orthogonal

will be called a Bamert-Hall array.

Orthogonal designs which give an overview of Baumert-Hall arrays are studied in [3] and [4].

We have

Theorem 1.4. (Baumert-Hal1). If there exist a Baumert-Hatz array of order $t$ and four williamson matrices of order $m$ then there exists an Hadamard matrix of order $4 \mathrm{mt}$.

Five years passed from the publication of the Baumert-llall array of order 3 until Lloyd welch [21] found his deceptively simple BaumertHall array of order 5 .

Shortly after Welch's matrix was discovered Jennifer Wallis [14] and Richard $J$. Turyn [12] independently announced that a construction of Goethals and Seidel [5] was important in finding Baumert-Hall arrays. Their theorem is

Theorem 1.5. (Goethals-Seidel [5]) If $X, Y, Z, W$ are square circulant $(1,-)$ matrices of order $t$, if $U=X$ - I is skew-symmetice, and if

$\operatorname{tin} i n$

$$
X X^{T}+Y Y^{T}+Z Z^{T}+W W^{T}=4 t I_{t}
$$

$$
G S=\left[\begin{array}{cccc}
X & Y R & Z R & W R \\
-Y R & X & -W^{T} R & Z^{T} R \\
-Z R & W^{T} R & X & -Y^{T} R \\
-W R & Z^{T} R & Y^{T} R & X
\end{array}\right]
$$

is a skew-Hadamard matrix of order $4 t$ when $R=\left(r_{i j}\right)$ of order $t$ is given by

$$
r_{i j}= \begin{cases}1, j=t+1-i, \\ 0 & \text { otherwise. }\end{cases}
$$

Wallis and Whiteman [15] showed how a similar matrix may be defined using an additive abelian group $G$. 
Theorem 1.6. (Wallis-Whiteman [15]). Let $Y, Y, W$ be type 1 (1, incidence matrices and $\mathrm{Z}$ be a type 2 (1,-) incidence matrix defined on the same abelian group of order $t$ (see [19] for definitions). If

then

$$
X X^{T}+Y Y^{T}+Z Z^{T}+W W^{T}=4 t I_{t}
$$

$$
H=\left[\begin{array}{cccc}
X & Y & Z & W \\
-Y^{T} & X^{T} & -W & Z \\
-Z & W^{T} & X & -Y^{T} \\
-W^{T} & -Z & Y & X^{T}
\end{array}\right]
$$

is an Hadamard matrix of order $4 \mathrm{t}$. Further if $\mathrm{X}-\mathrm{I}$ is skew, $\mathrm{H}$ is skew-Hadamard.

The following array is an example of a Baumert-Hall array of order 3 constructed using the Goethals-Seidel method.

\begin{tabular}{rrr|rrr|rrr|rrr}
$A$ & $B$ & $C$ & $B$ & $-C$ & $D$ & $C$ & $D$ & $-A$ & $D$ & $A$ & $-B$ \\
$C$ & $A$ & $B$ & $-C$ & $D$ & $B$ & $D$ & $-A$ & $C$ & $A$ & $-B$ & $D$ \\
$B$ & $C$ & $A$ & $D$ & $B$ & $-C$ & $-A$ & $C$ & $D$ & $-B$ & $D$ & $A$ \\
\hline$-B$ & $C$ & $-D$ & $A$ & $B$ & $C$ & $-D$ & $B$ & $-A$ & $C$ & $-A$ & $D$ \\
$C$ & $-D$ & $-B$ & $C$ & $A$ & $B$ & $B$ & $-A$ & $-D$ & $-A$ & $D$ & $C$ \\
$-D$ & $-B$ & $C$ & $B$ & $C$ & $A$ & $-A$ & $-D$ & $B$ & $D$ & $C$ & $-A$ \\
\hline$-C$ & $-D$ & $A$ & $D$ & $-B$ & $A$ & $A$ & $B$ & $C$ & $-B$ & $-D$ & $C$ \\
$-D$ & $A$ & $-C$ & $-B$ & $A$ & $D$ & $C$ & $A$ & $B$ & $-D$ & $C$ & $-B$ \\
$A$ & $-C$ & $-D$ & $A$ & $D$ & $-B$ & $B$ & $C$ & $A$ & $C$ & $-B$ & $-D$ \\
\hline$-D$ & $-A$ & $B$ & $-C$ & $A$ & $-D$ & $B$ & $D$ & $-C$ & $A$ & $B$ & $C$ \\
$-A$ & $B$ & $-D$ & $A$ & $-D$ & $-C$ & $D$ & $-C$ & $B$ & $C$ & $A$ & $B$ \\
$B$ & $-D$ & $-A$ & $-D$ & $-C$ & $A$ & $-C$ & $B$ & $D$ & $B$ & $C$ & $A$
\end{tabular}

Definition 1.7. Four type 1 (or circulant) $(0,1,-)$ matrices $x_{1}, x_{2}, x_{3}, x_{4}$ of order $t$ defined on the same abelian group royclic group) $G$ of order $t$ such that each of the $t^{2}$ positions is nonzero in precisely one of the $\mathrm{X}_{i}$ and satisfying

$$
x_{1} x_{1}^{T}+x_{2} x_{2}^{T}+x_{3} x_{3}^{T}+x_{4} x_{4}^{T}=t I_{t}
$$

will be called T-matrices. 
These matrices may be used to form Baume t-Hall arrays as follows:

Theorem 1.8. (Cooper-Wallis [2]). Suppose there exist four Tmatrices $\mathrm{X}_{1}, \mathrm{X}_{2}, \mathrm{X}_{3}, \mathrm{X}_{4}$ of order $t$. Further supiose that $\mathrm{A}, \mathrm{B}, \mathrm{C}$, D satisfy $\mathrm{MN}^{\mathrm{T}}=\mathrm{NM}^{\mathrm{T}}$ and let

$$
\begin{aligned}
& X=x_{1} \times A+x_{2} \times B+x_{3} \times C+x_{4} \times D, \\
& Y=x_{1} \times-B+x_{2} \times A+x_{3} \times D+x_{4} \times-C, \\
& Z=\left(x_{1} \times-C+x_{2} \times-D+x_{3} \times A+x_{4} \times B\right) R, \\
& W=x_{1} \times-D+x_{2} \times C+x_{3} \times-B+x_{4} \times A,
\end{aligned}
$$

with $R=\left(r_{i j}\right)$ defined on the elements of $G, g_{1}, g_{2}, \ldots, g_{t} b y$

$$
r_{\ell, j}= \begin{cases}1 & \text { if } g_{\ell}+g_{j}=0, \\ 0 & \text { otherwise: }\end{cases}
$$

Then $(t$ ) gives a Baumert-Hazl array of order $4 t$.

T-matrices may be easily shown to have the following properties:

Lema 1.9. Let $x_{1}, x_{2}, x_{3}, x_{4}$ be T-matrices of order $t$. Further let $x_{i}$ be the number of positive elements in each row and column of $x_{i}$ and $w_{i}$ the row sum (column sum) of each row (column) of $x_{i}$. Then
(a) $2\left(x_{1}+x_{2}+x_{3}+x_{4}\right)-\left(w_{1}+w_{2}+w_{3}+w_{4}\right)=t$,
(b) $w_{1}^{2}+w_{2}^{2}+w_{3}^{2}+w_{4}^{2}=t$.

\section{PREVIOUS CONSTRUCTION OF T-MATRICES}

In [2] and [8] T-matrices are constructed, ad hoc, for various small orders using guessing and a little cyclotomy.

Apparently, Richard J. Turyn has used circulant T-matrices $X_{1}=I, \quad X_{2}, \quad X_{3}, \quad X_{4}=0$ of order $t=2 a_{10} b_{2} c, a, b, c$ non-negative integers, to get the first infinite class of T-matrices. We have been able to construct these matrices having $(t-1) / 2$ non-zero entries in each row and column of $x_{2}$ and $x_{3}$. These matrices also exist for order 37 .

\section{A NEW CONSTRUCTTON USING T'-MATRICES}

Definition 3.1. Matrices (or Iinear combinations of matrices), $A_{i} \times B_{i}$, which may be used in the following array to form an liadamard 


$$
\left[\begin{array}{rrrr}
A_{1} \times B_{1} & A_{2} R \times B_{2} & A_{3} R \times B_{3} & A_{4} R \times B_{4} \\
-A_{2} R \times B_{2} & A_{1} \times B_{1} & A_{4}{ }^{T} R \times B_{4} & -A_{3}{ }^{T} R \times B_{3} \\
-A_{3} R \times B_{3} & -A_{4}{ }^{T} R \times B_{4} & A_{1} \times B_{1} & A_{2}{ }^{T} R \times B_{2} \\
-A_{4} R \times B_{4} & A_{3}{ }^{T} R \times B_{3} & -A_{2}{ }^{T} R \times B_{2} & A_{1} \times B_{1}
\end{array}\right]
$$

The $A_{i}$ is called the Goethals-Seidel part (GS-part) and the $B_{i}$ the Wilziamson part (W-part).

Theorem 3.2. Let $v \in\left\{1+2 \mathrm{a}_{10} \mathrm{~b}_{26} \mathrm{c}\right.$, a,b,c non-negative integers $\}$ $u\{37\}$. Suppose $2 v-1$ is a prime power $\equiv 1$ (mod 4); then there exist F-matrices of order $v(2 \mathrm{v}-1)$ and an Hadamard matrix of Goethals-Seidel type of order $4 \mathrm{v}(2 \mathrm{v}-1)$.

Proof. Let $I, x_{1}, x_{2}$, be the T-matrices of order $v$. Let $x$, $Y$ be the type $1(1,-1)$ incidence matrices of the $2-\{2 v-1 ; v-1 ; v-2\}$ supplementary difference sets described in [20, p.283]. Then

$$
\begin{gathered}
X^{T}=X, \quad Y^{T}=Y, \quad X J=Y J=-J, \quad X Y^{T}=Y X^{T}, \\
X X^{T}+Y Y^{T}=4 V I-2 J .
\end{gathered}
$$

Now choose

$$
\begin{aligned}
& A_{1} \times B_{1}=A=I \times J+\left(X_{2}+X_{3}\right) \times X, \\
& A_{2} \times B_{2}=B=I \times J-\left(X_{2}+X_{3}\right) \times Y, \\
& A_{3} \times B_{3}=C=I \times X-\left(X_{2}-X_{3}\right) \times Y, \\
& A_{4} \times B_{4}=D=I \times Y+\left(X_{2}-X_{3}\right) \times X .
\end{aligned}
$$

We write each of these in a form such as $A=A_{1} \times B_{1}$ even though in fact $A$ is a linear combination of such terms and use $A_{4}{ }^{T} \times B_{4}$ for

$$
R \times Y+\left(X_{2}-X_{3}\right)^{T} R \times X .
$$

Now we may easily check that $A, B, C$, and $D$ are the required F-matrices.

Example. Let $v=3$. The $2-\{5 ; 2 ; 1\}$ supplementary difference sets are $\{1,4\}$ and $\{2,3\} \bmod 5$ and forming the circulant $(1,-1)$ incidence matrices of these sets (where - is written for -1) we get 


$$
X=\left[\begin{array}{ccccc}
- & 1 & - & - & 1 \\
1 & - & 1 & - & - \\
- & 1 & - & 1 & - \\
- & - & 1 & - & 1 \\
1 & - & - & 1 & -
\end{array}\right] \text { and } Y=\left[\begin{array}{ccccc}
- & - & 1 & 1 & - \\
- & - & - & 1 & 1 \\
1 & - & - & - & 1 \\
1 & 1 & - & - & - \\
- & 1 & 1 & - & -
\end{array}\right] \text {. }
$$

Now for $v=3$ the T-matrices are

$$
\text { I, } X_{2}=\left[\begin{array}{lll}
0 & 1 & 0 \\
0 & 0 & 1 \\
1 & 0 & 0
\end{array}\right], \quad X_{3}=\left[\begin{array}{lll}
0 & 0 & 1 \\
1 & 0 & 0 \\
0 & 1 & 0
\end{array}\right]
$$

and so

$$
\begin{aligned}
& A_{1} \times B_{1}=\left[\begin{array}{rrr}
J & X & X \\
X & J & X \\
X & X & J
\end{array}\right], A_{2} \times B_{2}=\left[\begin{array}{rrr}
J & -Y & -Y \\
-Y & J & -Y \\
-Y & -Y & J
\end{array}\right], \\
& A_{3} \times B_{3}=\left[\begin{array}{rrr}
X & -Y & Y \\
Y & X & -Y \\
-Y & Y & X
\end{array}\right], A_{4} \times B_{4}=\left[\begin{array}{rrr}
Y & X & -X \\
-X & Y & X \\
X & -X & Y
\end{array}\right] .
\end{aligned}
$$

The required Hadamard matrix is

$$
\left[\begin{array}{rrrrrrrrrrrr}
J & X & X & J & -Y & -Y & X & -Y & Y & Y & X & -X \\
X & J & X & -Y & -Y & J & -Y & Y & X & X & -X & Y \\
X & X & J & -Y & J & -Y & Y & X & -Y & -X & Y & X \\
& & & & & & & & & & & \\
-J & Y & Y & J & X & X & Y & -X & X & -X & -Y & Y \\
Y & Y & -J & X & J & X & -X & X & Y & -Y & Y & -X \\
Y & -J & Y & X & X & J & X & Y & -X & Y & -X & -Y \\
& & & & & & & & & & & \\
-X & Y & -Y & -Y & X & -X & J & X & X & J & -Y & -Y \\
Y & -Y & -X & X & -X & -Y & X & J & X & -Y & -Y & J \\
-Y & -X & Y & -X & -Y & X & X & X & J & -Y & J & -Y \\
& & & & & & & & & & & \\
-Y & -X & X & X & Y & -Y & -J & Y & Y & J & X & X \\
-X & X & -Y & Y & -Y & X & Y & Y & -J & X & J & X \\
X & -Y & -X & -Y & X & Y & Y & -J & Y & X & X & J
\end{array}\right]
$$


This construction does not give new Hadamar natrices as williamson matrices are known for the orders given by the thorem.

4. CONSTRUCTION OF T-SETS FOR SMALL $t$

Definition 4.1. The set of four first rows $\mathrm{T}_{1}, \mathrm{~T}_{2}, \mathrm{~T}_{3}, \mathrm{~T}_{4}$ of four T-matrices $\mathrm{x}_{1}, \mathrm{x}_{2}, \mathrm{x}_{3}, \mathrm{x}_{4}$ witl be called a T-set.

To denote $\mathrm{T}$-sets, the element $j(j)$ in $\mathrm{T}_{i}$ indicates that the jth term $t_{i j}$ is $+1(-1)$; otherwise $t_{i j}=0$. Only the non-empty sets $T_{i}$ are listed, separated by slashes. Thus for $t=5,12 / 3 \overline{4} / 5$ denotes the $\mathrm{T}$-set

$$
\begin{aligned}
& \{11000\},\{001-0\},\{00001\},\{00000\} . \\
& \text { Obviously, a T-set is transformed into a T-set by a shift }
\end{aligned}
$$
$(j+j+b)$ or by a multiplier m relatively prime to $t(j \rightarrow m j)$, where the arithmetic is mod $t$. (These are essentially operations on supplementary difference sets.)

Now to find all T-sets for a fixed $t$, we consider all possible representations

$$
t=a_{1}^{2}+a_{2}^{2}+a_{3}^{2}+a_{4}^{2}=n_{1}+n_{2}+n_{3}+n_{4},
$$

where $T_{i}$ has $a_{i}+b_{i}+1^{\prime} s, b_{i}-1 ' s, t-n_{i} 0^{\prime} s$, and $n_{i}=a_{i}+2 b_{i} \geq a_{i}$. We search through all these possibilities to find T-sets.

The search is considerably simplified by the transformations noted above. For example, if $t=7=2^{2}+1^{2}+1^{2}+1^{2}$, the only possibilities for the $n_{i}$ are $\{4,1,1,1\}$ and $\{2,3,1,1\}$. Consider the latter case. We may choose the 3 -set first. There are $\left(\begin{array}{l}7 \\ 3\end{array}\right)=35$ of them, but by shifting we may assume that the 3 -set contains the element 1 , leaving only $\left(\begin{array}{l}6 \\ 2\end{array}\right)=15$ possibilities. But all of these can be transformed to one of the sets $\{1,2,3\}$ and $\{1,2,4\}$. Thus there are essentially only two cases to consider.

In the table we give complete lists of T-inequivalent T-sets of orders $t=3,5,7,9$. For each $t$ we list the values of $a_{1}, a_{2}, a_{3}, a_{4}$, then $n_{1}, n_{2}, n_{3}, n_{4}$ in $(\hat{3})$, then all T-inequivalent T-sets that occur with these parameters. [We note that most of the possible cases that do not in fact occur are ruled out by simple parity arguments. Only in a few cases was it necessary to test various assignments of +1 and -1 to rule out a case.] 


\section{Table of T-set:}

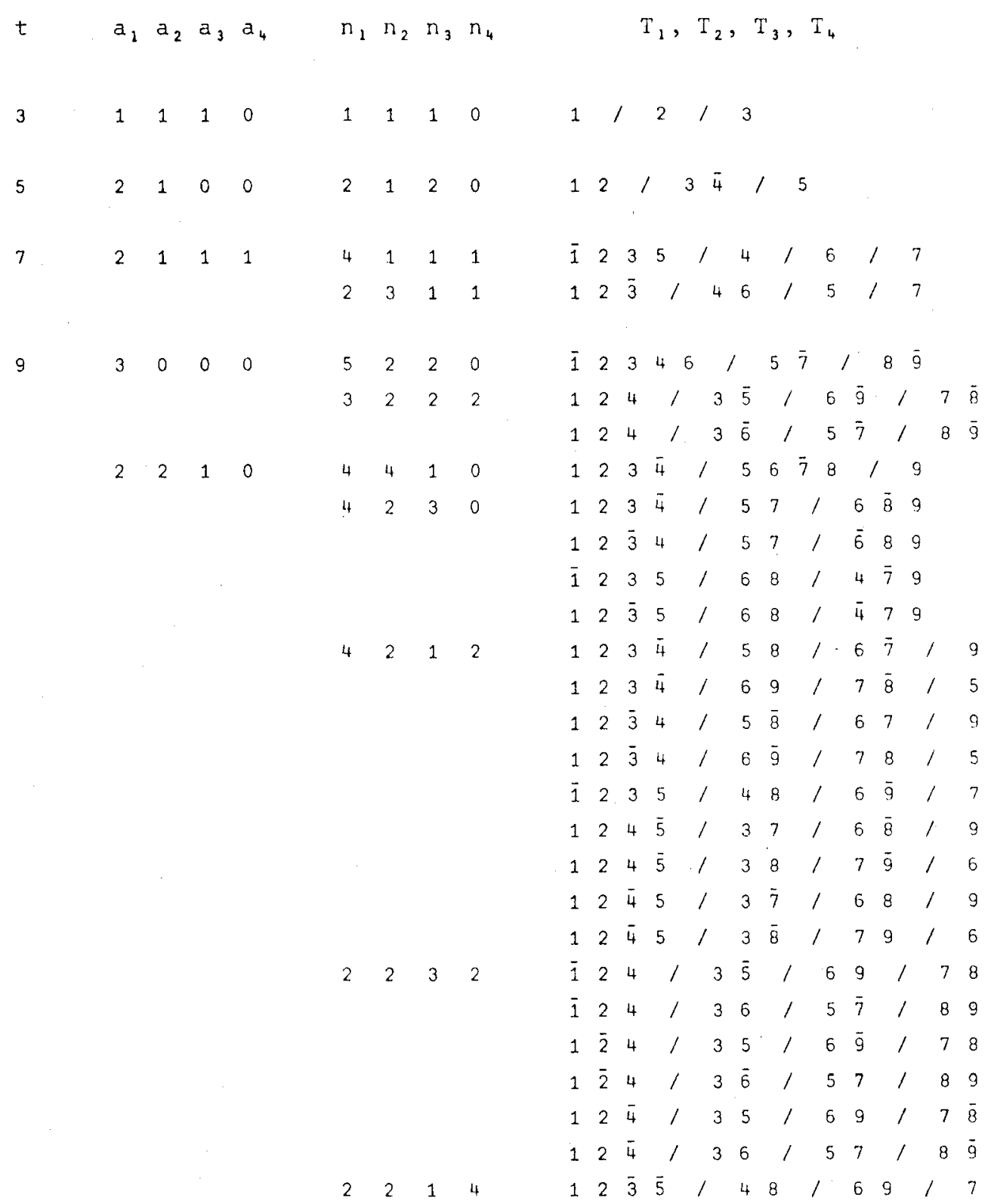


Note: for $t=9$ we list the T-inequivalut 2-sets,..,7-sets.

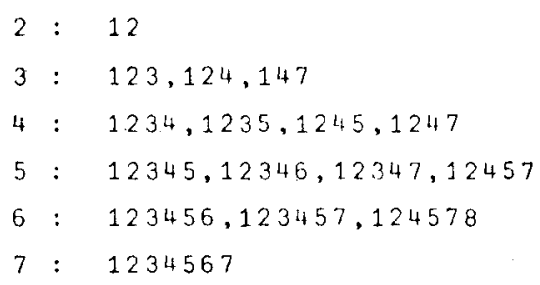

We also note the following remarkable occurrence. For each case with $t=9$ we inserted the matrices $x_{1}, x_{2}, x_{3}, x_{4}$ in the GoethalsSeidel array to obtain a $(0,1,-1)$ matrix $M$ satisfying $M^{T}=3 I_{36}$. The smith Normal form (see $[9, \mathrm{p} .44]$ ) was then calculated: necessarily there are $r 1^{\prime} s,(36-2 r) 3^{\prime} s$, and $r$ 9's. For each case corresponding to the decomposition $9=3^{2}$, we found $r=16$. For each case corresponding to $9=2^{2}+2^{2}+1^{2}$, we found $r=18$.

\section{SOME THOUGHTS ON EQUIVALENCE}

Definition 5.1. Two $(0,1,-1)$ matrices $X$ and $Y$ of order $t$ will be called T-equivalent if their first rows $X^{\prime}=\left\{x_{i}\right\}$ and $Y^{\prime}=\left\{y_{i}\right\}, i=1, \ldots, t$, given in the notation used above, can be obtained from one another by using multipliers and shifts, i.e. if $x_{i}=m_{i}+b(\bmod t)$, for integers $m, b$ and $i=1, \ldots, t$.

Definition 5.2. Two matrices $A$ and $B$, of order $n$ will be called H-equivalent or Hadamard equivalent if there exist $(0,1,-1)$ matrices $\mathrm{P}$ and $\mathrm{Q}$ with $|\operatorname{det} \mathrm{P}|=|\operatorname{det} \mathrm{Q}|=1$ such that

$$
B=P A Q \text {. }
$$

First we show that alteration of T-sets by shifting does not alter. Hadamard equivalence.

$$
\begin{aligned}
& \text { Let } T=\left(t_{i j}\right) \text { of order } n \text { be defined by } \\
& \qquad t_{12}=t_{23}=\ldots=t_{n-1, n}=t_{n, 1}=1
\end{aligned}
$$

and all other elements zero. Let $A, B, C, D$ be polynomials in $T$ and $S=T^{X}$ for some integer $x$. Let $R=\left(r_{i j}\right)$ be the matrix of order $n$ with

$$
r_{1, n}=r_{2, n-1}=\ldots=r_{n-1,2}=r_{n, 1}=1
$$

and all other elements zero. 
Then A, B, C, D, $S$ and their transpose'; pairwise comnute, $S^{T} R=R S, \quad R^{T}=R$ and $S S^{T}=I$.

Let

$$
P=\left[\begin{array}{cccc}
A & B R & C R & D R \\
-B R & A & D^{T} R & -C^{T} R \\
-C R & -D^{T} R & A & B^{T} R \\
-D R & C^{T} R & -B^{T} T_{R} & A
\end{array}\right]
$$

and

$$
Q=\left[\begin{array}{cccc}
A S & B S R & C S R & D S R \\
-B S R & A S & (D S)^{T} & -(C S)^{T} R \\
-C S R & -(D S)^{T} R & A S & (B S)^{T}{ }^{T} \\
-D S R & (C S)^{T} R & -(B S)^{T} & A S
\end{array}\right] \text {. }
$$

Then

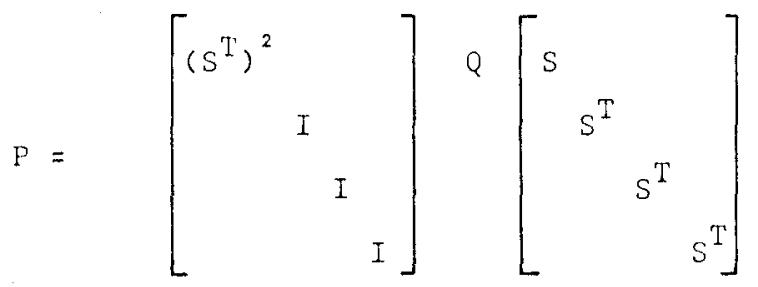

and so $P$ and $Q$ are Hadamard equivalent.

Also

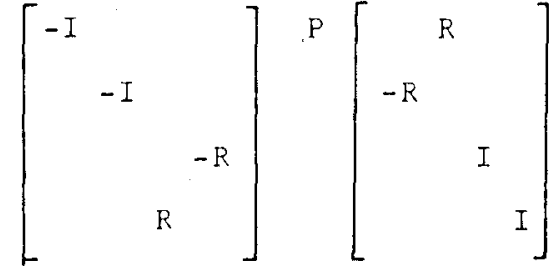

$$
\begin{aligned}
& =\left[\begin{array}{cccc}
-B & A R & C R & D R \\
-A R & -B & D^{T} R & -C^{T} R \\
-C R & -D^{T} R & -B & A^{T} R \\
-D R & C^{T} R & -A^{T} R & -B
\end{array}\right]
\end{aligned}
$$

which is the same as $P$ with $A$ and $B$ interchanged except that the interchanging of $A$ and $B$ has forced a change in sign of $B$. Niso 


$$
\begin{aligned}
& {\left[\begin{array}{ccc}
I & & \\
& -I & \\
& R & \\
& & -R
\end{array}\right]{ }^{P}\left[\begin{array}{cc}
2^{2}-R & \\
-R & \\
& -I
\end{array}\right]} \\
& =\left[\begin{array}{cccc}
B & A R & D R & C R \\
-A R & B & C^{T} & -D^{T} R \\
-D R & -C^{T} & B & A^{T} \\
-C R & D^{T} R & -A^{T} R & B
\end{array}\right],
\end{aligned}
$$

which resembles $P$ except that the interchanging of $A$, and $B$ has forced $C$ and $D$ to interchange too.

\section{UNANSWERED QUESTIONS ON EQUIVALENCE}

1. We noted for T-matrices of order 9 the decompositions into squares $9=3^{2}+0^{2}+0^{2}+0^{2}$ and $9=2^{2}+2^{2}+1^{2}+0^{2}$ gave T-inequivalent $T$-matrices and $Z$-inequivalent ( $P, Q$ of definition 5.2 have integer entries) and hence $H$-inequivalent weighing matrices $W(36,9)$. Do different decompositions into squares always give $z-$ inequivalent and hence H-inequivalent (i) weighing matrices (ii) Hadamard matrices (iii) Baumert-Hall arrays?

2. If the $X$ and $Y$ of $(*)$ are interchanged is the new Hadamard matrix or Baumert-Hall array H-equivalent to the old one? In section 5 it was observed that interchanging $X$ and $Y$ of $(*)$ either (i) induced $Z$ and $W$ to interchange or ( $i i) \quad Y$ to be replaced by $-Y$. Does this interchanging lead to H-inequivalence?

3. Let $X_{1}, X_{2}, X_{3}, X_{4}$ and $Y_{1}, Y_{2}, Y_{3}, Y_{4}$ be two sets of $T$ matrices of order $n$ corresponding to the same decomposition of $n$ into squares. Further suppose $X_{i}$ is not $T$-equivalent to $Y_{j}$ for any $i, j \in\{1,2,3,4\}$. Prove the Hadamard matrices and Baumert-Hall arrays formed from the $X_{i}$ and $Y_{i}, \quad i \in\{1,2,3,4\}$ are H-inequivalent. of 4. Let $x_{1}, x_{2}, x_{3}, x_{4}$ be T-matrices of order $n$. Does the use 


$$
\begin{aligned}
& X=x_{1} \times A+x_{2} \times B+x_{3} \times C+x_{4} \times D \\
& Y=x_{1} \times-B+x_{2} \times A+x_{3} \times-D+x_{4} \times C \\
& Z=\left(x_{1} \times-C+x_{2} \times D+x_{3} \times A+x_{4} \times-B\right) R \\
& W=x_{1} \times-D+x_{2} \times-C+x_{3} \times B+x_{4} \times \wedge
\end{aligned}
$$

in Theorem 1.7 instead of the $X, Y, Z, W$ given in the enunciation there lead to H-inequivalent Baumert-Hall arrays or Hadamard matrices?

\section{REFERENCES}

[1] L. D. Baumert and Marshall Hall, Jr., A new construction for Hadamard matrices, Bull. Amer. Math. Soc. 71 (1965) 169-170.

[2] Joan Cooper and Jennifer Wallis, A construction for Hadamard arrays, Bull. Austral. Math. Soc. 7 (1972) 269-278.

[3] A. V. Geramita, Joan Murphy Geramita and Jennifer Seberry Waliis, Orthogonal designs, Linear and Multilinear Algebra (to appear)

[4] A. V. Geramita and Jennifer Seberry Wallis, Orthogonal designs II, Aequationes Math (to appear).

[5] J. M. Goethals and J. J. Seidel, A skew-Hadamard matrix of order 36, J. Austral. Math. Soc. 11 (1970) 343-344.

[6] J. M. Goethals and J. J. Seidel, Orthogonal matrices with zero diagonal, Canad. J. Math. 19 (1967) 1001-1010.

[7] Marshall Hall, ur., Combinatorial Theory (Blaisdell, [Ginn and Co.], Waltham, Massachusetts, 1967).

[8] David C. Hunt and Jennifer Wallis, Cyclotomy, Hadamard arrays and supplementary difference sets, Proceedings of the second Manitoba Conference on Numerical Mathematics, Congressus Numerantium VII (1973) 351-381 (University of Manitoba, Winnipeg). 
[9] M. Marcus and H. Minc, A Survey of Matrix heory and Matrix Inequalities, (Allyn and Bacon, Boston, 1964).

[10] Edward Spence, Skew-Hadamard matrices of the Goethals-Seidel type, (to appear).

[11] Richard J. Turyn, An infinite class of Williamson matrices, J. Combinatorial Theory (Series A), 12 (1972) 319-321.

[12] Richard J. Turyn, The computation of certain Hadamard matrices, Notices Amer. Math. Soc. 20 (1973), A-2.

[13] Richard J. Turyn, Hadanard matrices, algebras, and composition theorems, Notices Amer. Math. Soc. 19 (1972), A-388.

[14] Jennifer Wallis, Hadamard matrices of order $28 \mathrm{~m}, 36 \mathrm{~m}$, and $44 \mathrm{~m}$, J. Combinatorial Theory (Series A), 15 (1973) 323-328.

[15] Jennifer Wallis and Albert Leon Whiteman, Some classes of Hadamard matrices with constant diagonal, Bulz. Austral. Math. Soc. $7(1972) 233-249$.

[16] Jennifer Wallis, Some matrices of Williamson type, Utilitas Math. 4 (1973) $147-154$.

[17] Jennifer Seberry Wallis, Williamson matrices of even order, Combinatorial Mathematics: Proceedings of Second Australian Conference (editor D.A. Holton), (Lecture Notes in Mathematics, Vol. 403, Springer-Verlag, Berlin-Heidelberg-New York, 1974.)

[18] Jennifer Seberry Wallis, Construction of Williamson type matrices, (to appear)

[19] Jennifer Seberry, Wallis, Recent advances in the construction of Hadamard matrices, Proceedings of the Fourth Southeastern Conference on Combinatorics, Graph Theory and Computing, Congressus Numerantium VIII (1974) 53-89 (University of Manitoba, Winnipers). 
[20] W. D. Wallis, Anne Fenfold Street, Jennifer Seberry Wallis, Combinatorics: Room Squares, Sum-free Sets, Hadamard Matrices, (Lecture Notes in Mathematics, Vol. 292, Springer-Verlag, Berlin-Heidelberg-New York, 1972)

[21] L. R. Welch, unpublished work.

[22] Albert Leon Whiteman, An infinite family of Hadamard matrices of Williamson type, $J$. Combinatorial Theory (Series A), (to appear)

[23] Albert Leon Whiteman, Williamson type matrices of order $2 q(q+1)$ (to appear).

[24] John Williamson, Hadamard's determinant theorem and the sum of four squares, Duke Math. J., 11 (1944) 65-81.

Department of Mathematics

State University of New York at Buffalo

Amherst, New York

U.S.A.

Department of Pure Mathematics

Institute of Advanced Studies

Australian National University

Canberra 